\title{
Wearing Comfort of Water Absobent Acrylic Fibers
}

\author{
By Kenji Arai, Members, TMSJ . \\ Acrylic Research Laboratory, Kanebo Hikone Plant, Shiga Pref. \\ Based on Journal of the Textile Machinery Society of Japan, Proceedings, Vol. 36, No. I, P.33-P.41 (1983-1)
}

\begin{abstract}
The physical properties of water absorbent porous acrylic fibers related to wearing comfort, i.e., water absorbency, drying, warmth retention, air permeability and frictional property have been compared with those of cotton and other fibers.

An investigation has shown that, compared to regular acrylic fibers, modified water absorbent acrylic fibers:

(1) Easily diffuse water and dry.

(2) Have a warmth retention intermediate between those of cotton and acrylic fibers.

(3) Have difficulty in losing their air permeability even when wet.

(4) Don't easily adhere to the skin even when wet.

The unique characteristics of water absorbent acrylic fibers listed above are a remarkable departure from conventional acrylic fibers as well as from other synthetic fibers.

This means an unlimited number of end uses for water absorbent acrylic fibers where moisture absorbing properties are important for comfort or fiber performance.
\end{abstract}

\section{Introduction}

One of the inherent characteristics of the so-called three major synthetic fibers is hydrophobicity or a lack of hygroscopicity and water absorbing capability. While these qualities help them to display a variety of features such as being quick drying, not shrinking and stretching in water, and long lasting, this undisputedly limits the further development of their end-uses.

Natural fibers such as cotton, wool, and silk offer wearing comfort because they have a higher water absorbing capacity than synthetic fibers, and because they sufficiently absorb perspiration. Whereas, synthetic fibers may be less commercially valuable than natural fibers since they are not hygroscopic, having virtually no moisture absorbing quality. For wearing comfort, it is desirable for isolated free water to exist only within the fibers, not between the woven yarns of the fabric. To ascertain the amount of water within fibers, the equation in item 3-2 is used to calculate water content at the saturation point. The calculation shows that wool contains $30 \%$ water at the saturation point, and cotton $41 \%$, while the regular acrylic fiber contains only $6 \%$. See Fig. 3 . Without water absorbing capability, underwear, socks, sportswear, and similar items will inevitably cause the wearer discomfort as the perspiration from the skin will condense on and stick to fibers, thus allowing the fabric to cling to the skin. This will chill the skin, eventually resulting in decreased temperature regulating functions.

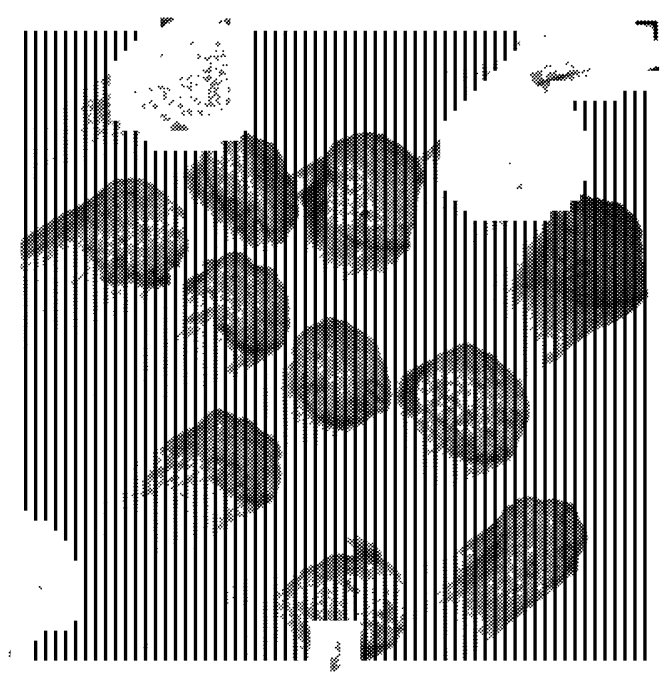

Fig. 1 Microscopically enlarged cross-sectional view of Kanebo "Lumiza"

Therefore, constant efforts have been made by synthetic fiber manufacturers to impart moisture and water absorbing properties to synthetic fibers. Roughly classified, these efforts have been directed toward three methods ${ }^{[1,2]}$ such as making the polymer itself water-affinitive, rendering the fiber surface water-affinitive through a post-spinning treatment ${ }^{[3]}$, and modifying the fiber itself so that it is multi-porous. Of the three, the post-spinning treatment has been actively ap- 
plied to nylon, polyester, and acrylic fibers by either synthetic fiber manufacturers or finishers. The imparting of water absorbing properties to synthetic fiber by making the fiber itself multi-porous has been exemplified by Bayer A.G. for Dunova, Kanebo, Ltd. for Lumiza, Mitsubishi Rayon Co. for Swift-K, Asahi Chemical Industry Co. for Solcy, and Nihon Exlan for Selipar ${ }^{[4,7]}$. A microscopically enlarged cross-sectional view of Kanebo Lumiza is shown in Fig. 1, which has fine multi-pores extending throughout the fiber.

Broadly defined, the term, water-absorbing property, includes hygroscopicity as well. Here in this technical report, the writer wants its meaning limited to the absorption of water in its liquid phase. In a sampling of studies that determine the relationship between water absorbency and the resultant comfort, it is shown, for instance, in A. Suzuki's report ${ }^{[5]}$ in which he compares the relationship between the hydrophobic synthetic and hydrophilic natural fibers. In addition, how the water absorbing capacity, imparted to the synthetic fiber, will affect its physical properties and resultant comfort, and also how such a capacity will compare with that of cotton are analyzed ${ }^{[7]}$ as detailed by W. Körner, et al. ${ }^{[6]}$, who has used Dunova for the analysis. In particular, this report describes wear-comfort-related areas in reference to Kanebo Lumiza as well as to newly developed test procedures, the detail of which will be described later in this report.

\section{Test Fabrics}

Table 1 shows specifications of knit fabrics (smooth) used for testing various physical properties, while Table 2 also shows those of woven fabrics (plain) used for testing physical properties. The air permeability was tested only on woven fabrics, and other physical properties were tested on knitted fabrics.

Before testing physical properties, the foreign matter in Kanebo Lumiza knit fabrics was extracted with ethanol for three hours, and then washed with a non-ionic surfactant (Noigen EA-120, $15 \mathrm{~g} /$ liter) for 50 minutes at $40^{\circ} \mathrm{C}$. Cotton knits were scoured for 60 minutes at $100^{\circ} \mathrm{C}$ in a $2 \% \mathrm{NaOH}$ bath with the ratio of cotton to solution at 1:50, and then subjected to the physical properties test. Other knits underwent the same extraction process for 1.5-4 hours, washed with a non-ionic surfactant, and their physical properties tested. Of woven fabrics, cotton fabrics were alkali-scoured under the same conditions as for knitted fabrics, and then subjected to a physical properties test, while other woven fabrics were washed for 30 minutes at $85^{\circ} \mathrm{C}\left(30^{\circ} \mathrm{C}\right.$ for only wool) in a $0.2 \%$ non-ionic surfactant solution (Sunmol BK) with the ratio of the test fabric to solution at $1: 50$, and then physical properties were tested. The water absorbing acrylic fibers mentioned in this report are meant as porous acrylic fibers with water absorbing capability.

\section{Test Results and Comments}

3.1 Water Absorbency and Water Transferring Properties Either perspiration or water absorbing capabilities of fibers can be broadly classified into three functions:

a) Water absorbing speed of the fiber related to how

Table 1 Specifications of knit fabrics used for testing physical properties

\begin{tabular}{|c|c|c|c|c|}
\hline & Kanebo"Lumiza" & Regular acrylics & Cotton & Wool \\
\hline Fineness & $1.5 \mathrm{~d}$ & $1.5 \mathrm{~d}$ & $1.4 \mathrm{~d}$ & $19.3 \mu$ \\
\hline Count & $1 / 60$ & $1 / 64$ & $36 / 1(1 / 61)$ & $1 / 60$ \\
\hline Construction & \multicolumn{4}{|c|}{ Smooth } \\
\hline Thickness (mm) & 1.17 & 0.78 & 0.77 & 1.16 \\
\hline Normal weight $(\mathrm{g} / \mathrm{sq} \cdot \mathrm{m})$ & 207 & 190 & 192 & 206 \\
\hline Volume of air porosity $(\%)$ & 79.9 & 79.2 & 84.1 & $86: 5$ \\
\hline Apparent density $(\mathrm{g} / \mathrm{cu} \cdot \mathrm{cm})$ & 0.18 & 0.24 & 0.25 & 0.18 \\
\hline
\end{tabular}

Table 2 Specifications of woven fabrics used for testing physical properties

\begin{tabular}{l|l|l|l|l}
\hline & Kanebo "Lumiza" & Regular acrylics & Cotton & Wool \\
\hline Fineness & $1.5 \mathrm{~d}$ & $1.5 \mathrm{~d}$ & $1.4 \mathrm{~d}$ & $19.3 \mu$ \\
\hline Count & $2 / 60$ & $2 / 64$ & $36 / 2(2 / 61)$ & $2 / 60$ \\
\hline Construction & \multicolumn{3}{|c}{ Plain } \\
\hline Thickness $(\mathrm{mm})$ & 0.43 & 0.40 & 0.35 & 0.47 \\
\hline Normal weight $(\mathrm{g} / \mathrm{sq} \cdot \mathrm{m})$ & 212 & 207 & 219 & 205 \\
\hline Volume of air porosity $(\%)$ & 44.0 & 55.7 & 59.0 & 66.4 \\
\hline Apparent density $(\mathrm{g} / \mathrm{cu} \cdot \mathrm{cm})$ & 0.49 & 0.52 & 0.63 & 0.44 \\
\hline
\end{tabular}


quickly it can absorb perspiration, b) water transferring capacity of the fiber concerned with how far a given amount of water can spread within a given time, and c) ability of the fiber to hold water under certain conditions. These three requirements can be simply stated as water absorbtion speed, water diffusion property, and water retention quality. Any fiber, meeting these three requirements, can be said to have achieved a major breakthrough in imparting comfort to resultant garments. In order for the fiber to fully meet other comfort requirements, it must satisfy a few additional requirements such as perspiration evaporating capabilities, and freedom from clinging as well as from feeling wet and cold.

Three test methods such as those for determining wettability of water droplets, water elevation velocity, and specimen precipitation by soaking are basically important for evaluating the water pick-up speed. In connection with this, the microporously modified version of acrylic fiber attains the water absorbing speed of less than one second when tested for water droplet wettability and the water elevation height of more than $130 \mathrm{~mm}$ when tested for elevation velocity. Particularly, the modified version of acrylic fiber has shown a greater difference in water elevation than regular acrylic fibers when tested. The writer has related the readings in Table 3 to wearing comfort as follows: The water absorbing velocity obtained from the wettability method will be a good determinant of the perspiration absorbing functions for dry underwear. Whereas this does not apply to underwear that is already wet or when actually worn. In this case, the water elevation determining method applies. The less time it takes a dry fabric to absorb water (within one second), the better it is. One of the characteristic features of a microporously modified acrylic fiber is its quick water diffusion performance as proved by the water elevation determining method. It has higher water elevating capacity than cotton, wool, and regular acrylic fibers, the test specimens of which were prepared under the same conditions. See Fig. 2. This is due to the fact that acrylic fibers are inherently wettable ${ }^{[8]}$ and that, in the case of microporously modified acrylic fibers, they have a

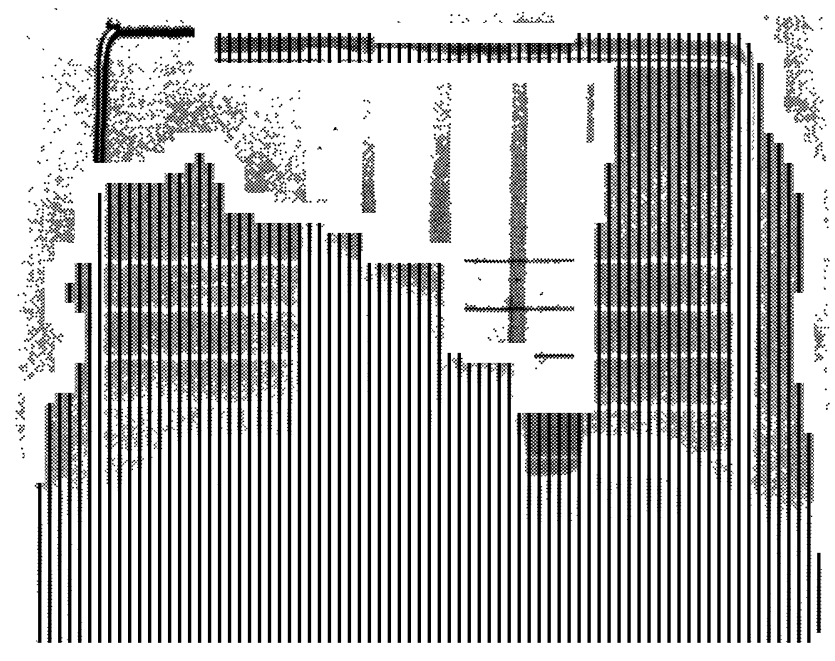

Fig. 2 Water elevation by capillary action of different fibers.

built-in capillary action.

The multi-porous acrylic fiber thus allows the water on its surface to diffuse along the fiber and also to go into the fiber simultaneously. This provides extremely quick water absorption; the water movement will take place both on the fiber surface and within the fiber, water invariably reaching an otherwise inaccessible inner portion of the fiber. Whereas, the regular acrylic fiber is said to be virtually incapable of allowing water to enter inside the fiber, water movement and absorption occuring only on its surface. Cotton accomplishes water absorption by allowing it to diffuse into fine hollows. Particularly in cotton it swells remarkably immediately after water absorption, a trait that causes a decrease in its air permeability. However, acrylic fibers are practically free from this characteristic; in that microporously modified acrylic fibers, at least Kanebo Lumiza, does not create the swelling phenomenon. This will be described in more detail later in this report. As might be expected, vertically or horizontally positioned multi-porous acrylic fabrics as well as two-layered fabrics will allow the water to prop-

Table 3 Water absorbing speeds

\begin{tabular}{l|l|l|l}
\hline & $\begin{array}{l}\text { Water elevation } \\
\text { determining } \\
\text { method (mm) }\end{array}$ & $\begin{array}{l}\text { Wettability } \\
\text { method by water } \\
\text { droplet (Second) }\end{array}$ & $\begin{array}{l}\text { Specimen precipitation } \\
\text { method by soaking } \\
\text { (Second) }\end{array}$ \\
\hline Kanebo "Lumiza" & 137 & Instantly & 5 \\
\hline Regular acrylics & 87 & Instantly & 5 \\
\hline Cotton & 120 & Instantly & 4 \\
\hline Wool & 0 & 10 min. or over & 10 min. or over \\
\hline Remarks & \multicolumn{2}{|c}{ JIS L 1018-1977 } & $\begin{array}{l}\text { Standard specified } \\
\text { by Daimaru Department } \\
\text { Store }\end{array}$ \\
\hline
\end{tabular}


agate equally fast; from up to down in the case of a vertical position, horizontally in the case of a horizontal position, and from upper to lower layers in the case of a two-layered fabric. Such unique behavior of multi-porous acrylic fibers serve to diffuse the moisture of a garment over a wide area even when it is soaking wet. The wide diffusion means less residual water content per unit area of a garment; provides comfort because of a minimal wet feeling, and prompts correct body temperature regulation (the regulation, in this particular case, means the decreasing of the temperature of the innermost layer of a garment) because of an enlarged sweat evaporation area and an increased sweat evaporation amount. The quick moisture evaporating capability permits a garment to approach a dried state as nearly and as quickly as possible in a short period of time even if it is soaking wet. This fact shows how multi-porous water absorbing acrylic garments provide wearing comfort. Whether the temperature is hot, dry, wet or cold, people doing strenuous work or playing sports well perspire. What I have dealt with thus far in this section cannot be completed without working on related matters such as water transferal and retention as well as air transferal and heat retention. I would like to address these matters in another report.

\subsection{Water Retention}

In the case of the multi-porous water absorbing acrylic fiber, water will first diffuse into minute pores, and be held there. The water thus retained there cannot be removed by mechanical power, such as centrifugal force, but will be transfer to the surface upon drying. Unless microporous

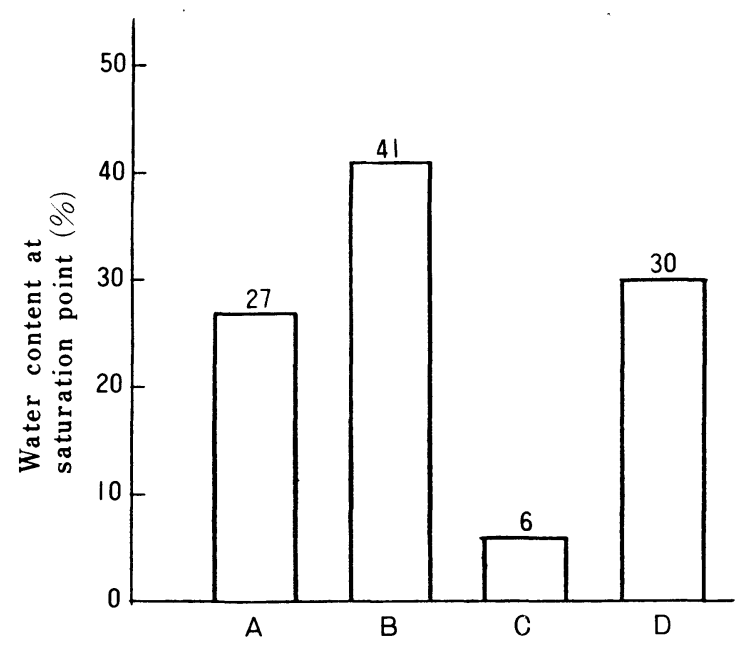

Fig. 3 Water content at saturated point of different fibers
A: Kanebo "Lumiza"
B: Cotton
C: Regular acrylics
D: Wool

fibers contain too much water in their pores, the isolated water existing between fiber spaces is negligibly little: This enables the fiber to display its characteristic features such as freedom from clinging, as well as from not having a cold and wet feeling.

Water retention (water content at the saturation point) is evaluated as follows. Well-opened test fibers are first immersed in water, centrifuged for 10 minutes by a force of $1,000 \mathrm{~g}$, and then calculated by the following equation:

Water content at the saturation point $(\%)=W-W_{0} /$ $W_{0} \times 100$

wherein

$W=$ Weight of test fiber obtained in the preparation procedure for the above specimen

$W_{0}=$ Weight of test fiber $\mathrm{W}$, which is further conditioned under standard atmospheric conditions.

Figure 3 shows the water content of different fibers at their saturation point.

\subsection{Drying Properties}

Figure 4 shows variation in the water content obtained

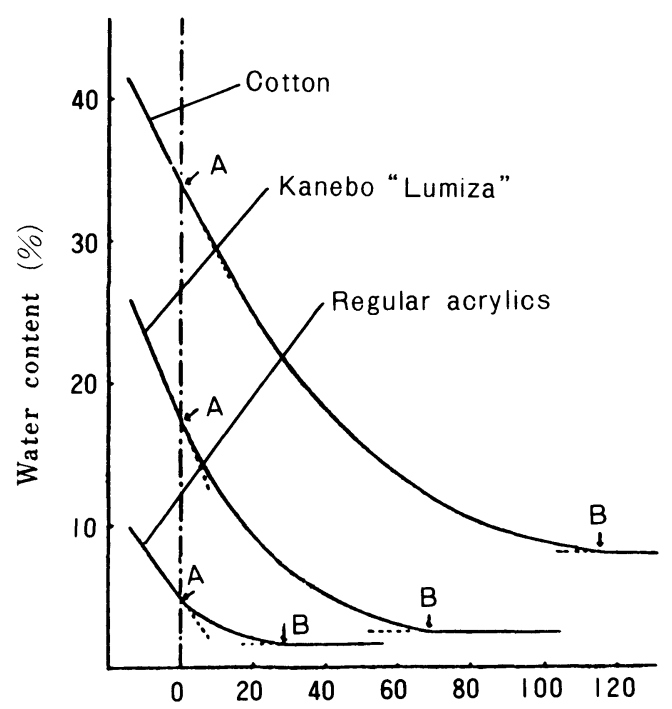

Fig. 4 Water content variation over the passage of time

from drying tests in which three different knit specimens were subjected. Figure 5 shows curves showing the drying properties of four different knit specimens. Both figures are prepared from identical drying test results. As is seen in Fig. 4, three different curves representing three different knit specimens originate from beyond the starting point of the drying test or from a constant rate period, because this will help understand the water content history of the specimens in relation to their drying at a falling rate period that starts from A, critical moisture content, and ends with $B$, end of drying at the falling rate period. It can be seen from Figs. 4 




Fig. 5 Curves showing drying characteristics.

and 5 that water absorbent acrylic fibers have a shorter falling rate period than those of cotton and wool: The former ends the falling rate period $44 \%$ faster than cotton. Such water absorbent behavior in acrylic fiber is considered to be more essential and useful than anything else for underwear and sportswear that will possibly undergo repeated perspiration and evaporation cycles.

Quick drying capability is one of the important factors for clothing that comes in direct contact with perspiration. Unlike cellulosic fibers which are chemically bonded with hydrophilic groups, multiporous acrylic fibers physically retain water merely in its capillaries, thus displaying quick evaporation capabilities.

Drying tests for knit specimens were made in a temperature and moisture controlled laboratory, in which there was a tempers ture variation from 18.0 to $18.8^{\circ} \mathrm{C}$ and a relative humidity variation from 60 to $68 \%$ during four readings taken from each test knitted fabric. The wind velocity there was not controlled.

Knit specimens (smooth), $10 \mathrm{~cm} \times 10 \mathrm{~cm}$, were immersed in pure water for ample time, centrifuged, dried on a net placed near a direct-reading balance, and weighed on the balance every one minute for the first $\mathbf{3 0}$ minutes, every two minutes for the second 30 minutes and every five minutes for the third 30 minutes. They were dried to an absolute dry condition after reaching a constant weight, calculated for the absolute dry weight, and moisture content determined. After the completion of the above test procedures, the readings thus obtained were plotted against the moisture releasing velocity per unit time $(\mathrm{mg} / \mathrm{min})$ as well as against the water content (Wf) at an interval of 6-7 minutes. The results are shown in Fig. 5 to identify the values of critical moisture content and the consistency ${ }^{[9,10]}$ of the drying rates for each test fabric at a constant rate period.

\subsection{Wetting and Heat Retention}

One of the most important factors that determine the heat retention of an apparel fabric is fabric thickness and air content. Measuring thick fabrics for their heat retention shows that heat retention will increase ${ }^{[11]}$ as thickness increases. The heat retention of the apparel fabric also depends on how much it can keep the still air in it. Given that the thickness is the same, how densely the fabric is woven will determine the heat retention in a certain range of thicknesses. In the case of multiporous fiber, it entraps still air within itself which can less easily flow out, thus apparently contributing to heat retention. However, this contribution is small, if any, because ordinary clothing will entrap as much as $80 \%$ of air, but, multiporous fibers themselves comprising only $20 \%$ of such clothing.

Since the clothing which one puts on is a mixture of fibers, air and perspiration, the matter dealing with heat retention and conductivity must be studied with relation to moisture. So, the writer will brief readers on two methods of measuring the heat retention while supplying water to the water absorbent and regular acrylic knit specimens as well as to cotton knit specimens. One is a hot plate method, and the other a cylindrical method. ${ }^{[12]}$

The heat retention is determined by $\mathrm{L} 1018$ A-method or constant temperature method specified by the Japanese Industrial Standard Method, after each specimen was predampened to different water content up to $60 \%$.

Three pieces of knit specimens, $25 \mathrm{~cm} \times 25 \mathrm{~cm}$, are conditioned as uniformly as possible to a prescribed water content with an atomizer, sewn together in three layers using cotton yarn at five spots, and then subjected to heat retention tests. Since the constant temperature method is one which determines the heat lost through the specimen wthin 2 hours, no additional water supply was made during the test.

As is shown in Fig. 6, the water absorbent acrylic fabric displays higher heat retention than cotton and regular acrylic fabrics even when wet. The constant temperature method, which does not dampen the specimen during the test except at the initial stage, makes the specimen as nearly alike as the damp clothing which one has on in an airconditioned room after active sports or after working under a hot sun. The initially dampened constant temperature method gives test results of heat retention depending on how the specimen will be dried.

The writer will now describe the test results of heat retention obtained from an intermittent water supply method. Figs. 7(a) and 7(b) show test results of heat retention in power consumption that compare the heat retention be- 


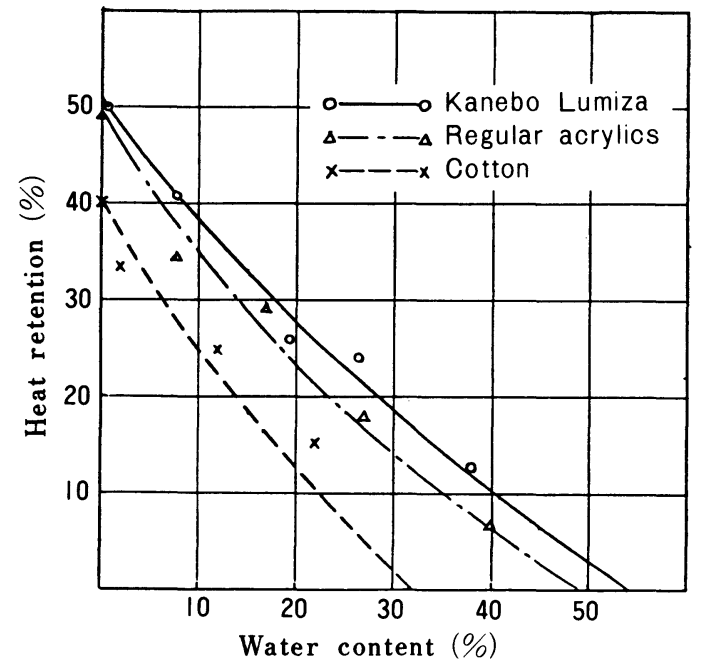

Fig. 6 Heat retention of different wet fabrics measured by JIS constant temperature method

tween specimens using a constant temperature cylinder method ${ }^{[12]}$ by T. Wada. Actually, this method is intended to identify what the constant temperature method (JIS L 1018 A-method) failed to ascertain in heat retention tests. Briefly, Wada's method determines the power consumption $(\mathrm{W} / \mathrm{hr})$ used up by a test specimen to maintain the same temperature for 60 minutes, the test piece of which is wrapped around a constant temperature warm water-filled perforated cylinder, from which a small amount of water intermittently oozes out through the test piece.

Heat retention behaviors of pieces when initially wet were also measured for comparison.

The atmosphere in the test room is maintained at $20 \pm 0.5^{\circ} \mathrm{C}$ and $65 \pm 3 \% \mathrm{RH}$, and a heat retention testing unit is installed there, the copper cylinder beeing filled with $36.5^{\circ} \mathrm{C}$ water, its middle portion having five $0.5 \mathrm{~mm}$ dia. apertures for oozing the water out. A test piece, $21 \mathrm{~cm} \times 31 \mathrm{~cm}$, is wrapped around the cylinder. The test results in Fig. 7(a) are those obtained from dry test pieces, while those in Fig. 7(b) are those obtained from the intermittent water supply procedure.

The constant temperature cylinder method is capable of simulating the human body perspiration mechanism, hence the relationship between the damp clothing and heat retention. When 1 milliliter of water is released onto a $651 \mathrm{sq} \cdot \mathrm{cm}$ specimen at the release rate of 1 milliliter/10 minutes and repeated six times to release a total of 6 milliliters of water onto the specimen, the total amount thus transferred comes to 92 grams $/ 100 \mathrm{sq} \cdot \mathrm{cm} / \mathrm{hr}$; the amount is more perspiration than $74 \mathrm{grams} / 100 \mathrm{sq} \cdot \mathrm{cm} / \mathrm{hr}$ which a person weighing $65 \mathrm{~kg}$ will secrete while leading an ordinary life, but less perspiration $124 \mathrm{grams} / 100 \mathrm{sq} \cdot \mathrm{cm} / \mathrm{hr}$ which a manual worker usually loses while doing his work. ${ }^{[13]}$ In each simulated test, however,

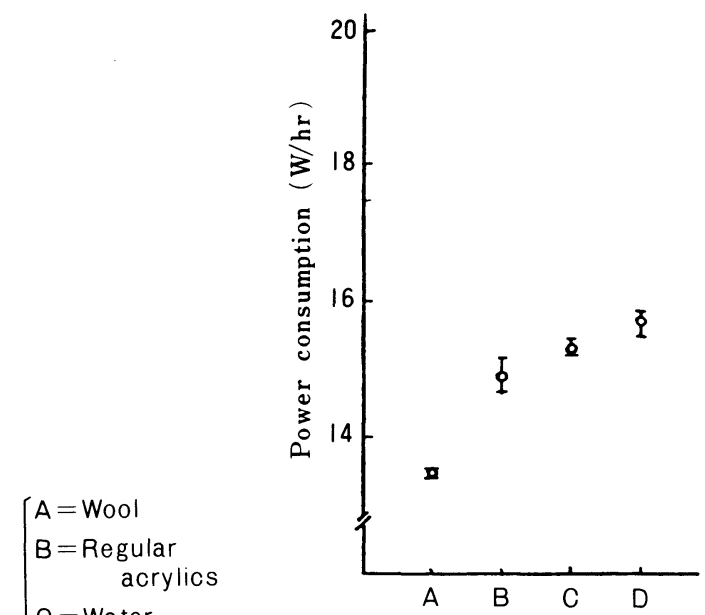

$C=$ Water
$\quad$ absorbent
acrylics
$D=$ Cotton

(a) Dry test specimen

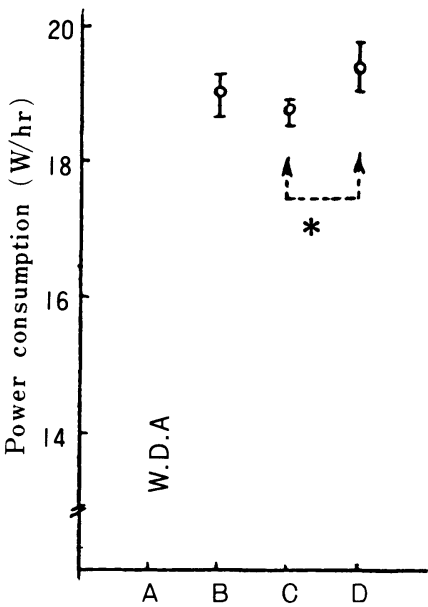

(b) Intermittent water supply method

Fig. 7 Heat retention in power consumption

the total amount of transferred water, $92 \mathrm{grams} / 100 \mathrm{sq} \cdot \mathrm{cm} /$ $\mathrm{hr}$, corresponds to the actual perspiration of $184 \mathrm{grams} / 100$ $\mathrm{sq} \cdot \mathrm{cm} / \mathrm{hr}$ which is twice that of 92 grams, because the water exuding from the perforation could not reach the edge portions of the test piece, wetting only half its area. The perspiration of 184 grams approaches the perspiration of 237-373 grams $/ 100 \mathrm{sq} \cdot \mathrm{cm} / \mathrm{hr}$ which a person loses in walking an hour under the midsummer sun when temperature is $32-36^{\circ} \mathrm{C}$.

With their weight and other specifications kept the same, the four different knit specimens were subjected to the heat retention tests. The results are as follows: In the case of dry test specimens, they showed a slight difference in power consumption (heat retention). See Fig. 7(a). Figure 7(b) shows the power consumed by the four different fibers during intermittent perspiration. There is a significant difference in power consumption between the water absorbent acrylic fiber 
and cotton. The water absorbent acrylic fiber that is wet provides higher heat retention than does the cotton.

Tests were made to see how the varying residual water content of three different fibers would relate to power consumption (heat consumption), and their results are shown in Fig. 8. Regular acrylic fibers almost completely dried during the 60 minute drying test, reducing heat retention only by heat lost through evaporation, while cotton and water absorbent acrylic fibers ended the 60 minute drying test with some residual water, thus resulting in less power consumption. The dotted line in Fig. 8 shows the heat lost through $6 \mathrm{cc}$ water evaporation.

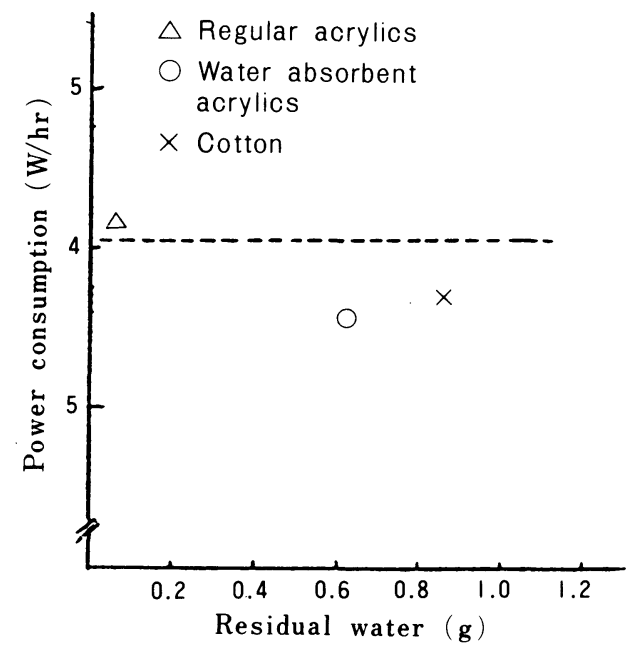

Fig. 8 Relationship between residual water and power consumption for dry and wet specimens

The quicker the underwear dries, the faster it recovers the wearing comfort, while at the same time this drying speed, will deprive the human body of heat through moisture evaporation.

To simulate the human wearers who are each wearing water absorbent, regular acrylic and cotton underwear, which is soaked in perspiration, and who have lost body heat through evaporation, each knit specimen is wrapped around the cylinder for heat retention and wetted with 6 milliliters of water to read the continuous heat lost through water evaporation. The test results are shown in Fig. 9. As is seen in Fig. 9 , the regular acrylic knit specimen consumes more power at first, using less water later. The cotton knit specimen consumes power more moderately with the consumption rate being less than the regular acrylic knit specimen. The water absorbent acrylic knit specimen is between the two.

To sum up the foregoing test results, the heat retaining properties found in the wet water absorbent acrylic fiber are such that they will not allow the human body to lose as much heat as the regular acrylics do, nor will they rob the

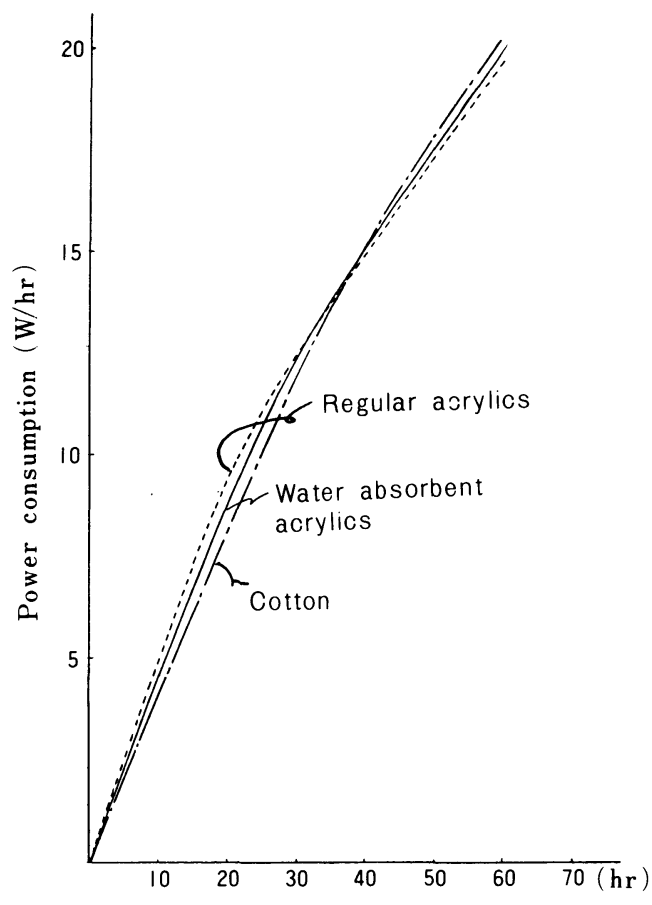

Fig. 9 Integrated power consumption over the passage of time

human body of heat for the length of time that cotton does.

\subsection{Wettability and Air Permeability}

Inherently, wettability and air permeability largely depend on the weaving and knitting construction than on the types of fibers. Other determinants of wettability and air permeability attributable to the fiber itself include crimp, cross sectional shapes of fiber, mode of twisting, and the resultant apparent yarn size and bulkiness. In actual wearing, however, the clothing is subjected to constant air streams in and out, depending on cold and hot weather, on windy and calm days, and on the intensity of physical exercise. Also added to the effect of air permeability is the perspiration which may involve repeated wetting and drying, as well as the ambient temperature and shrinkage in washing.

In the number of factors that affect the air permeability as mentioned above, the writer indentified only the effect of air permeability on perspiration in the water absorbent acrylic fibers developed by Kanebo. Figure 10 shows how the air permeability varies as the test fabric gradually increases its water content from zero $\%$. The air permeability tests were conducted on somewhat densely woven fabrics containing $50 \%$ air, to help learn the effect of air permeability on fiber. Air permeability was tested on a Frazile tester.

The test results are shown in Fig. 10. The air permeability of cotton fabrics decreases continuously as their water content increases, while there is no decrease in air permeability of the water absorbent acrylic fiber fabrics even when they 


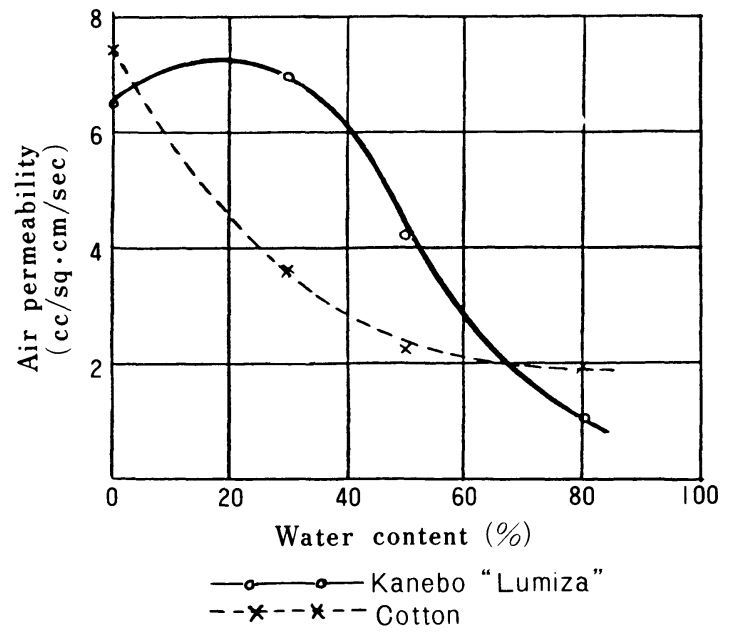

Fig. 10 Air permeability of wet fabric


(a) Cotton

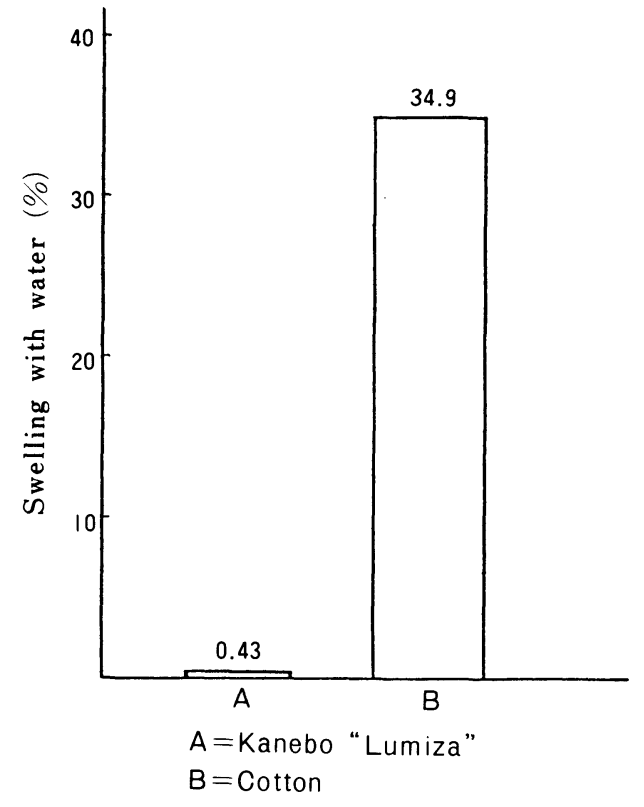

Fig. 12 Swelling with water

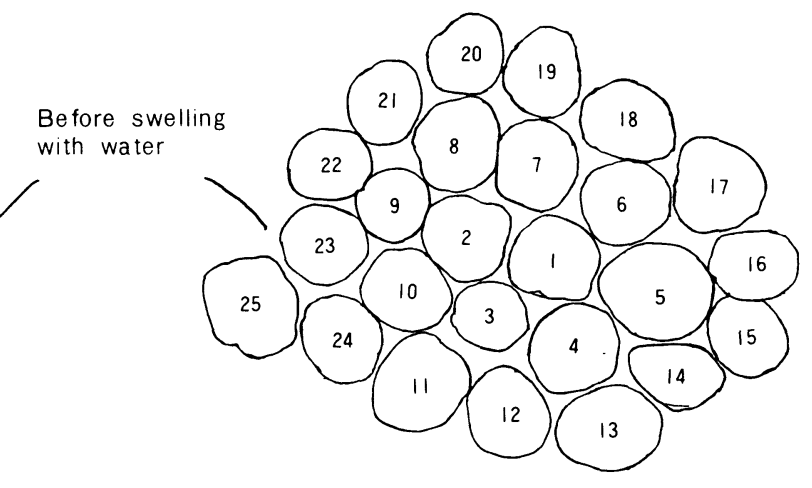

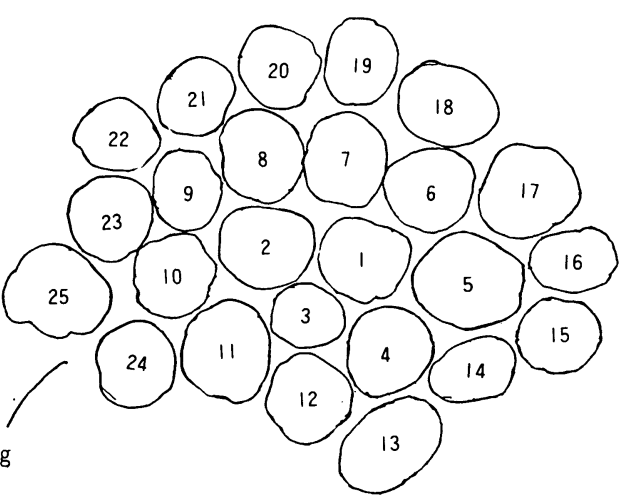

(b) Water absorbent acrylics

Fig. 11 Microscopically enlarged cross sectional view 
absorb $10-15 \%$ water, though their air permeability decreases when they absorb more water.

Thus water absorbent acrylic fiber loses air permeability and wearing comfort less easily even after it absorbs perspiration. Such distinctive characteristics are due to the fact that cotton swells and increases its size as it absorbs water, while the water absorbent acrylic fiber, except when it absorbs a large amount of water, absorbs water in its capillaries, not in its fiber-to-fiber spaces. Figure 11(a) shows microscopically enlarged cross sectional view of cotton, and Figure 11(b) shows water absorbent acrylic fiber, each of which is water-swollen. Figure 12 shows the percentage of swelling with water in cotton and Kanebo Lumiza.

\subsection{Clinging}

When wearing clothing and sportswear, particularly underwear that comes in contact with the skin, it is customary first to check their hand. They might also often be checked for their touch either after washing or when dampened by perspiration. Learning how a wet fabric changes its function, particularly its frictional properties in relation to its dry state, is an important matter that cannot be overlooked in dealing with underwear and sportswear fabrics. This is also true of the water absorbent acrylic fabric. The wet absorbent acrylic fabric should be compared with only cotton wet fabric for its frictional properties, because the two are different from each other in their water absorbing mechanisms and speed as well as in their mode of water retention, although they belong to the same category of water absorbent fibers.

The frictional properties of clothing mentioned in the above mean specifically static and dynamic frictions that arise between wet skin and underwear, being a good determinant of the clinging magnitude. To simulate conditions similar to wet fabric clinging to the skin, three methods were employed. The first method is to draw a test specimen horizontally over a resin plate surface to read the static frictional coefficient of the specimen. The second method is to read the fiber/fiber friction with a Rothschild friction meter. The third method or Suzuki's method ${ }^{[14]}$, is to read the frictional resistance that increases with a test specimen being pulled over a polypropylene film surface that has a drop of water added to it. See Fig. 14. More specifically, the first method that determines the static frictional coefficient is described as follows:

A $30 \mathrm{~cm} \times 20 \mathrm{~cm}$ test specimen, placed on a smooth resin plate surface, is applied a 2,500 gram load to read the force (f) which is perpendicularly exerting on the specimen, and horizontally drawn at the rate of $100 \mathrm{~mm} / \mathrm{second}$ to read the coefficient of static friction $(W g)$ that arises. The static frictional coefficient is worked out by the following equation.

Static frictional coefficient $\mu=W g / f$ wherein $g=$ gram.

In testing the static frictional coefficient, cotton fabric and Kanebo Lumiza piled fabric are used whose yarn counts

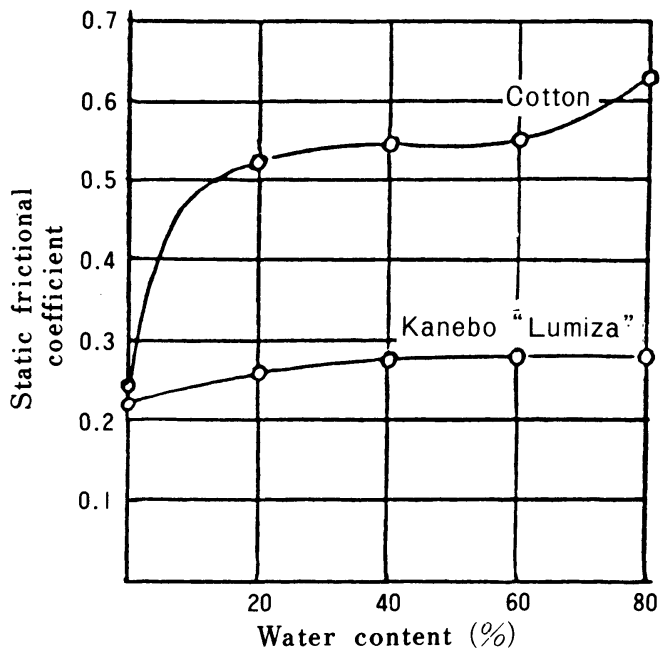

Fig. 13 Relationship between water content and static friction

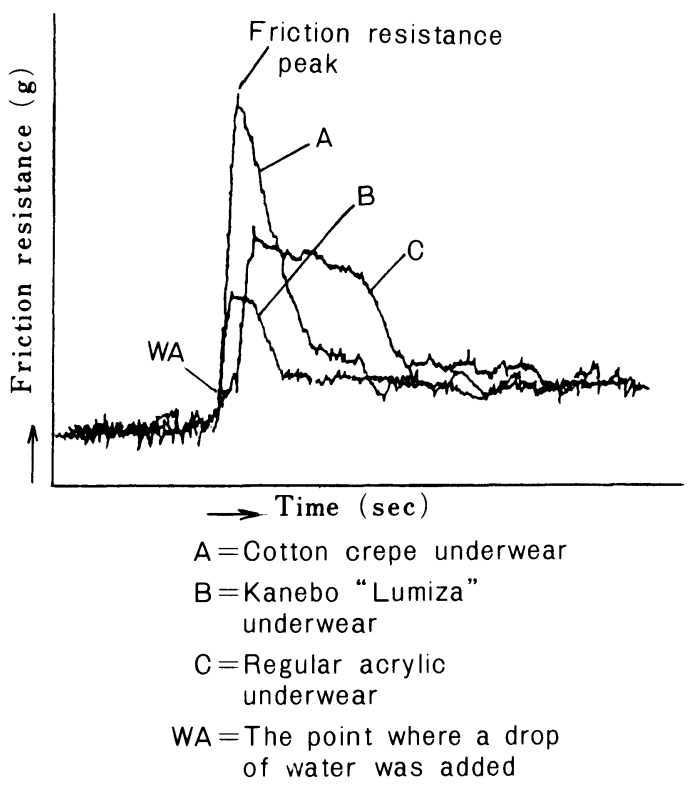

Fig. 14 Friction resistance that increases with time

and pile lengths are $20 / 2$ and $3 \mathrm{~mm}$. Tset results are given in Fig. 13.

The second method determines the yarn-to-yarn friction: Two yarns, cotton yarn 30/2 and Kanebo Lumiza 2/48, are used. See Fig. 15. The initial tension is kept at 10 grams $\left(T_{1}\right)$. A specimen is brought into contact with another specimen and moved at the rate of $2 \mathrm{~mm} / \mathrm{min}$ to read the resultant tension $T_{2}$, thus friction in both dry and wet states can be ascertained. The test results are listed in Table 4. The third method is as described below.

(1) A $5 \mathrm{~cm} \times 12 \mathrm{~cm}$ test strip is pulled over a water repel- 
Table 4 Yarn-to-yarn friction

\begin{tabular}{c|c|c|c|} 
& \multicolumn{3}{|c}{ Yarn-to-yarn friction (g) } \\
\hline & When dry & When wet & Wet/dry ratio \\
\cline { 2 - 4 } Kanebo & & & \\
"Lumiza" & 19.0 & 20.5 & 1.08 \\
Cotton (A) & 21.5 & 34.0 & 1.58 \\
Cotton (B) & 19.0 & 30.0 & 1.58 \\
\hline
\end{tabular}

lent finished resin film surface.

(2) A drop of water is released between the test strip and the film while the strip is still sliding.

(3) The slide resistance before and after the waterdrop release are read with a strain gauge, the slide resistance that changes with the time being read with a recorder.

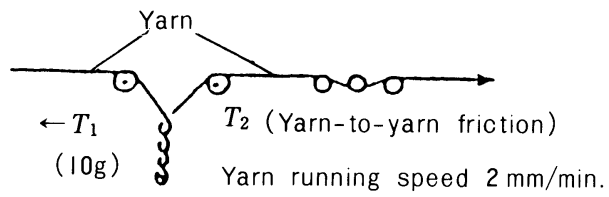

Fig. 15 Method of measuring yarn-to-yarn friction

The slide resistance tests were made on five $5 \mathrm{~cm} \times 12 \mathrm{~cm}$ test strips at the speed of $20 \mathrm{~mm} /$ minute with 100 microliter water being released. A 25 microliter syringe was used to release the waterdrop. As the Fig. 14 shows, the slide resistance that rapidly increases the moment the waterdrop is released is less with the water absorbent acrylic specimen than with other specimens. The attenuation length is also shorter.

\section{Conclusion}

A number of physical properties that are considered to represent the wearing comfort and performance of garments made using multi-porous water absorbent acrylic fibers are measured. Readings thus obtained are compared with those of cotton, wool and regular acrylic fibers, the comparison enabling the writer to conclude that the water absorbent acrylic fibers:

(1) Easily diffuse water and dry.

(2) Have a warmth retention intermediate between those of cotton and acrylic fibers.

(3) Have difficulty in losing their air permeability even when wet.

(4) Don't easily adhere to the skin even when wet.

\section{References}

[1] Tsunetaka Matsumoto; Kasen-Geppo, 29, 55 (1976-2).

[2] Takashi Harada; J. Soc. Fib. Sci. Tech., Japan, 32, P589 (1979-12).

[3] Norio Tanaka; Sensyoku-Kogyo, 30, 23 (1982-1).

[4] Kenji Arai; J. Soc. Fib. Sci. Tech., Japan, 37, P-105 (1981-2).

[5] Atsushi Suzuki; Reports on Natural Science, Dept. Educ. Nagasaki Univ. 207 (1981) No. 32.

[6] W. Körner, G. Blankenstein, P. Dorsch, U. Reinehr; Chemiefasern, 29, 452 (1979).

[7] Takeshi Fukui, Teruo Sekiguchi; J. Soc. Fib. Sci. Tech., Japan, 36, P-313 (1980).

[8] Haruo 'Sanuki; J. Japan, Res. Ass. Text. End-Uses, 20, 244 (1979-7).

[9] Toshinari Nakajima; Midori Shindoh, J. Soc. Fib. Sci. Tech., Japan, 20, T-347 (1981-9).

[10] Masako Niwa, Noriko Takada, Nobuko Tokaji; $J$. Japan, Res. Ass. Text. End-Uses, 3, 191 (1965-4).

[11] G. J. Morris; J. Text. Inst., T449 (1953).

[12] Tominari Wada, Harumi Kofukada, J. Japan, Res. Ass. Text. End-Uses, 21, 24 (1980-1).

[13] Osamu Yuge; Hygienics on clothing (HIFUKU-EISEI$G A K U)$ p. 30, Asakura Shoten (1980).

[14] Atsushi Suzuki, Michiyasu Ohira, J. Text. Mach. Soc. Japan, 26, T217 (1973-12). 